\title{
Mm CO observation of the old nova NQ Vul
}

\author{
J. S. Albinson \& A. Evans \\ Department of Physics, University of Keele, Keele, Staffordshire, ST5 5BG, \\ UNITED KINGDOM
}

\begin{abstract}
We have observed the old nova NQ Vul in the $\mathrm{J}=2 \rightarrow 1$ rotational transition of ${ }^{12} \mathrm{CO}$ at $230.5 \mathrm{GHz}$ at the University of Texas Millimetre Wave Observatory ${ }^{1}$. The spectrum shows narrow features which clearly arise in the local interstellar medium. However these local features are superimposed on a broad feature which peaks at $\sim 63 \mathrm{mK}$. This feature is centred on velocity $V_{L S R} \simeq 26 \pm 9 \mathrm{~km} \mathrm{~s}^{-1}$-consistent with that expected for NQ Vul-and has FWHM $80 \pm 20 \mathrm{~km}$ $\mathrm{s}^{-1}$. The peak antenna temperature corresponds to an integrated flux of $3.2 \times 10^{-15} \mathrm{erg} \mathrm{s}^{-1} \mathrm{~cm}^{-2}$.

Assuming LTE and a distance of $1.2 \mathrm{kpc}$ the mass of $\mathrm{CO}$ is $\sim 10^{-6} M_{\odot}$ if the line is optically thin. The CO mass is comparable with the total mass ejected in 1976 (4) so the CO we detect at millimetre wavelengths has nothing to do with the 1976 outburst. If the $\mathrm{CO} / \mathrm{H}$ ratio in the emitting material is similar to that in the interstellar medium (2), the total mass is $\sim 0.6 M_{\odot}$.

The CO mass rules out an origin both in the 1976 outburst and in the post-outburst phase: the CO must have originated in material ejected by the NQ Vul system prior to the 1976 outburst. There are two possibilities. First, the $\mathrm{CO}$ may have formed in material accumulated following a large number of nova outbursts. Second, the CO may have been present, or formed, in material ejected by the NQ Vul system during a previous evolutionary phase. The deduced mass is comparable to the mass of CO seen around planetary nebulae (3); the outflow velocity $\left(\sim 40 \mathrm{~km} \mathrm{~s}^{-1}\right)$ would also be in line with this interpretation.

In either case, the above mass estimate of $0.6 M_{\odot}$ (based on the interstellar $\mathrm{CO} / \mathrm{H}$ ratio) is likely to be an upper limit as we would expect an enhancement of heavy elements in any ejected material. A determination of ${ }^{12} \mathrm{C} /{ }^{13} \mathrm{C}$ and other isotopic ratios would be valuable to pin down the origin of the $\mathrm{CO}$.
\end{abstract}

The full text of this paper has been published in ref. 1 .

\section{References}

1. Albinson, J. S. \& Evans, A., 1989. Mon. Not. R. astr. Soc., in press.

2. Duley, W. W. \& Williams, D. A., 1984. Interstellar Chemistry, Academic Press, Oxford.

3. Masson, C. R., Cheung, K. W., Berge, G. L., Claussen, M. I., Heiligman, G. M., Leighton, R. B., Lo, K. Y., Moffet, A. T., Phillips, T. G., Sargent, A. I., Scott, S. L. \& Woody, D. P., 1985. Astrophys. J., 292, 464.

4. Ney, E. P. \& Hatfield. B. F., 1978. Astrophys. J., 219, L111.

\footnotetext{
${ }^{1}$ The Texas Millimetre Wave Observatory is supported by National Science Foundation Grant AST 8815801 and the the W. M. Keck Foundation.
} 